\title{
Energy Spectrum of Ejected Electrons of H(2P) Ionization by Electrons in Coplanar Asymmetric Geometry
}

\author{
Sunil Dhar*, Nurun Nahar \\ Department of Mathematics, Chittagong University of Engineering and Technology, Chittagong, Bangladesh
}

Email address:

sdhar@cuet.ac.bd (S. Dhar), nurun_nahar_ctg@yahoo.com (N. Nahar)

To cite this article:

Sunil Dhar, Nurun Nahar. Energy Spectrum of Ejected Electrons of H(2P) Ionization by Electrons in Coplanar Asymmetric Geometry. American Journal of Modern Physics. Vol. 4, No. 3, 2015, pp. 132-137. doi: 10.11648/j.ajmp.20150403.15

\begin{abstract}
We have investigated the energy spectrum of ejected electrons of hydrogen atoms ionization from metastable $2 \mathrm{P}$ state by electrons in coplanar asymmetric geometry. A multiple scattering theory of Das and Seal is applied. The present results show very interesting binary peak features. As well as a significant qualitative agreement with hydrogenic ground state results is obtained. New theoretical results and experimental verification for such treatment in hydrogenic metastable states by electrons will be interesting.
\end{abstract}

Keywords: Electron, Cross Sections, Ionization, Scattering

\section{Introduction}

The theoretical and experimental study in electron atom ionization collision on differential cross sections has become increasingly interesting over four decades for relativistic [1-5] as well as non-relativistic [6-17] energies. Various theoretical models applying differential kinematical conditions have been vastly used for experimental measurements. Ehrhardt et al. [5] have investigated this more successfully for different energies of the incident electrons from very low to high energies. The study of energy spectrum of ejected electrons could be very interesting.

Mott and Massey [18] had done integrated cross section results which did not show much interesting results. Schüle and Nakel [19] presented experimental investigation of the energy spectrum of ejected electrons for ionization of medium-heavy atoms by relativistic electrons. However, Das and Dhar [20] have investigated energy spectrum of ejected electrons for hydrogenic ground state ionization by electrons in the non-relativistic energy region. To the best of our knowledge there is no such study, for hydrogenic metastable $2 \mathrm{P}$ states ionization by electrons both theoretically and experimentally, reported in the literature, in the non-relativistic domain. Here we have studied the energy spectrum for hydrogenic metastable $2 \mathrm{P}$ state at $250 \mathrm{eV}$ and $500 \mathrm{eV}$ incident energies. Here we also use the earlier method of Das and Seal [21]. This theory [21] has been successfully used in the study of energy spectrum of ejected electrons for ionization of ground state hydrogen atoms
[20] and medium-heavy atoms by relativistic electrons [22-23]. Recently Dhar and Nahar [24-25] calculated triple differential cross sections for ionization of metastable 2P-State hydrogen atoms by electron impact for coplanar asymmetric geometry using the multiple scattering theory of Das and Seal [21].

The present study will be added for the significant contribution to the future theoretical results and experimental set up for the study of energy spectrum of ejected electrons from metastable hydrogen atoms by electrons. Lewis integral [26] has been used in the present study for analytical calculation.

\section{Theory}

Here we have applied the multiple scattering theory of Das and Seal [21] which is discussed here, briefly. The direct T-matrix element for ionization of hydrogen atoms by electrons may be written as

$$
T_{f i}=\left\langle\phi_{f}^{(-)}\left(\bar{r}_{1}, \bar{r}_{2}\right)\left|V_{i}\left(\bar{r}_{1}, \bar{r}_{2}\right)\right| \varphi_{i}\left(\bar{r}_{1}, \bar{r}_{2}\right)\right\rangle
$$

Here the perturbation potential $V_{i}\left(\bar{r}_{1}, \bar{r}_{2}\right)$ is given by

$$
V_{i}\left(\bar{r}_{1}, \bar{r}_{2}\right)=\frac{1}{r_{12}}-\frac{Z}{r_{2}}
$$

for hydrogen atom nuclear charge $(Z)=1, r_{1}$ and $r_{2}$ are the distances of the two electrons from the nucleus and $r_{12}$ is the distance between the two electrons. 
The initial channel unperturbed wave function is

$$
\Phi_{i}\left(\bar{r}_{1}, \bar{r}_{2}\right)=\frac{e^{i \bar{p}_{i} \cdot \bar{r}_{2}}}{(2 \pi)^{3 / 2}} \varphi_{2 p}\left(\overline{r_{1}}\right)
$$

i.e.

$$
\Phi_{i}\left(\bar{r}_{1}, \bar{r}_{2}\right)=\frac{e^{\bar{p}_{i} \cdot \bar{F}_{2}}}{8 \sqrt{2} \pi^{2}} r_{1} \cos \theta e^{-r_{i} \lambda_{1}}
$$

where

$$
\varphi_{2 p}\left(\bar{r}_{1}\right)=\sqrt{\frac{1}{32 \pi}} r_{1} \cos \theta e^{-r_{1} / 2}=\sqrt{\frac{1}{32 \pi}} r_{1} \cos \theta e^{-\lambda_{1} r_{1}},\left[\lambda_{1}=\frac{1}{2}\right]
$$

is the hydrogenic $2 \mathrm{P}$-state wave function, $\bar{p}_{i}$ is the incident electron momentum, and $\psi_{f}^{(-)}\left(\bar{r}_{1}, \bar{r}_{2}\right)$ is the final three-particle scattering state wave function with the electrons being in the continuum with momenta $\bar{p}_{1}, \bar{p}_{2}$. Co-ordinates of the two electrons are taken to be $\bar{r}_{1}$ and $\bar{r}_{2}$.

Here $\psi_{f}^{(-)}\left(\bar{r}_{1}, \bar{r}_{2}\right)$ is approximate wave function and is given by [21]

$$
\begin{gathered}
\psi_{f}^{(-)}\left(\bar{r}_{1}, \bar{r}_{2}\right)=N\left(\bar{p}_{1}, \bar{p}_{2}\right)\left[\varphi_{\bar{p}_{1}}^{(-)}\left(\bar{r}_{1}\right) e^{i \bar{p}_{2} \cdot \bar{r}_{2}}\right. \\
\left.+\varphi_{\bar{p}_{2}}^{(-)}\left(\bar{r}_{2}\right) e^{\overline{\bar{p}_{1}} \cdot \bar{r}_{1}}+\varphi_{p}^{(-)}(\bar{r}) e^{i \bar{P} \cdot \bar{R}}-2 e^{i \bar{p}_{1} \cdot \bar{r}_{1}+\bar{p}_{2} \cdot \bar{r}_{2}}\right] /(2 \pi)^{3}
\end{gathered}
$$

where $\bar{r}=\frac{\bar{r}_{1}-\bar{r}_{2}}{2}, \bar{R}=\bar{r}_{1}+\bar{r}_{2}$,

$$
\bar{p}=\left(\bar{p}_{2}-\bar{p}_{1}\right), \bar{P}=\bar{p}_{2}+\bar{p}_{1}
$$

The scattering amplitude may be written as

$$
f\left(\bar{p}_{1}, \bar{p}_{2}\right)=N\left(\bar{p}_{1}, \bar{p}_{2}\right)\left[f_{e T}+f_{P T}+f_{P e}-2 f_{P W B}\right]
$$

where $f_{e T}, f_{P T}, f_{P e}$ and $f_{P W B}$ are the amplitudes corresponding to the four terms of (4).

The normalization constant $N\left(\bar{p}_{1}, \bar{p}_{2}\right)$ is given by

$$
\begin{gathered}
\left|N\left(\bar{p}_{1}, \bar{p}_{2}\right)\right|^{-2}=\mid 7-2\left[\lambda_{1}+\lambda_{2}+\lambda_{3}\right]-\left[\frac{2}{\lambda_{1}}+\frac{2}{\lambda_{2}}+\frac{2}{\lambda_{3}}\right] \\
+\left[\frac{\lambda_{1}}{\lambda_{2}}+\frac{\lambda_{1}}{\lambda_{3}}+\frac{\lambda_{2}}{\lambda_{1}}+\frac{\lambda_{2}}{\lambda_{3}}+\frac{\lambda_{3}}{\lambda_{1}}+\frac{\lambda_{3}}{\lambda_{2}}\right] \mid
\end{gathered}
$$

Here

$$
\begin{gathered}
\lambda_{1}=e^{\pi \alpha_{1} / 2} \Gamma\left(1-i \alpha_{1}\right), \text { where } \alpha_{1}=1 / p_{1}, \\
\lambda_{2}=e^{\pi \alpha_{2} / 2} \Gamma\left(1-i \alpha_{2}\right), \text { where } \alpha_{2}=1 / p_{2}, \\
\lambda_{3}=e^{\pi \alpha / 2} \Gamma(1-i \alpha), \text { where. }
\end{gathered}
$$

And $\phi_{\bar{q}}^{(-)}(\bar{r})$ is the Coulomb wave function and is given by

$$
\phi_{\bar{q}}^{(-)}(\bar{r})=e^{\pi \alpha / 2} \Gamma(1+i \alpha) e^{i q . \bar{r}} F_{1}(-i \alpha, 1,-i[q r+\bar{q} \cdot \bar{r}]) .
$$

Now applying (2), (3) and (4) in (1), we get

$$
T_{f i}=T_{B}+T_{B^{\prime}}+T_{i}-2 T_{P B}
$$

where

$$
\begin{aligned}
T_{B} & =\left\langle\phi_{\bar{p}_{1}}^{(-)}\left(\bar{r}_{1}\right) e^{i \bar{p}_{2} \cdot \bar{r}_{2}}\left|V_{i}\right| \Phi_{i}\left(\bar{r}_{1}, \bar{r}_{2}\right)\right\rangle \\
T_{B^{\prime}} & =\left\langle\phi_{\bar{p}_{2}}^{(-)}\left(\bar{r}_{2}\right) e^{i \bar{p}_{1} \cdot \bar{r}_{1}}\left|V_{i}\right| \Phi_{i}\left(\bar{r}_{1}, \bar{r}_{2}\right)\right\rangle \\
T_{i} & =\left\langle\phi_{\bar{p}}^{(-)}(\bar{r}) e^{i \bar{P} \cdot \bar{R}}\left|V_{i}\right| \Phi_{i}\left(\bar{r}_{1}, \bar{r}_{2}\right)\right\rangle \\
T_{P B} & =\left\langle e^{i \bar{p}_{1 . \overline{1}_{1}+\bar{p}_{2} \cdot \bar{r}_{2}}}\left|V_{i}\right| \Phi_{i}\left(\bar{r}_{1}, \bar{r}_{2}\right)\right\rangle
\end{aligned}
$$

The direct scattering amplitude $f\left(\bar{p}_{1}, \bar{p}_{2}\right)$ is then determined from

$$
f\left(\bar{p}_{1}, \bar{p}_{2}\right)=-(2 \pi)^{2} T_{f i}
$$

The exchange amplitude is approximated by

$$
g\left(\bar{p}_{1}, \bar{p}_{2}\right)=f\left(\bar{p}_{2}, \bar{p}_{1}\right)
$$

After analytical calculation using the Lewis integral [26], the triple differential cross sections (TDCS) with exchange effects is finally given by,

$$
\frac{d^{3} \sigma}{d \Omega_{1} d \Omega_{2} d E_{1}}=\frac{p_{1} p_{2}}{p_{i}}\left[\frac{3}{4}|f-g|^{2}+\frac{1}{4}|f+g|^{2}\right]
$$

where, $\Omega_{1}$ and $\Omega_{2}$ are solid angles, $E_{1}$ is the ejected energy.

Calculation has been performed for the hydrogenic metastable $2 \mathrm{P}$ state for $250 \mathrm{eV}$ and $500 \mathrm{eV}$ incident electron energy, for scattering in a plane with fixed valuesof $\theta_{1}, \theta_{2}$ and for scattering values of the energy of the scattered electron.

\section{Results and Discussion}

Triple differential cross sections (TDCS) results for the energy spectrum of ejected electrons of hydrogen atoms in metastable 2P state are presented in Figs. 1-6 for the incident energy $250 \mathrm{eV}$ and in Figs. 7-10 for the incident energy $500 \mathrm{eV}$. The results are compared with the hydrogenic ground state results of Das and Dhar [20]. The ejected energy $E_{1}$ is considered in the range $5 \mathrm{eV}-100 \mathrm{eV}$ for different combinations of angles of the scattered and ejected electrons.

Firstly, in the Fig. 1, the incident energy is taken as $250 \mathrm{eV}$, the scattering angle is $\theta_{2}=-5^{0}$ and the ejected angle is 
$\theta_{1}=40^{\circ}$. We note that the energy spectrum for our present results show similar peak features as the compared results [20]. But the peak position is shifted to higher ejected energy about $E_{1}=72 \mathrm{eV}$ which was $40 \mathrm{eV}$ in the compared results [20]. Also, it is very interesting to notice that the peak height for the present results seems to be identical as the compared results [20] whereas no peak appears in the first Born results. Although the ejected angle is increased as $\theta_{1}=60^{\circ}$ (Fig. 2), the present and the first Born results show same behavior as that of Fig. 1 but the peak value in the present results is increased slightly.

In the Fig. 3, the present results display an interesting wave feature which upper peak value remain same as the previous results but the lower peak value is about $40 \mathrm{eV}$ ejected energy whereas the compared results [20] show an upper peak at $28 \mathrm{eV}$ ejected energy.

The energy spectrum of scattered electron of hydrogen atoms in metastable $2 \mathrm{P}$ state by $250 \mathrm{eV}$ for the scattered angle $\theta_{2}=-10^{\circ}$ and the ejected angle $\theta_{1}=40^{\circ}$ (Fig. 4) is somewhat different. Here the peak height of the present results is decreased whereas there was a very sharp peak in the compared results [20] at about $28 \mathrm{eV}$ ejected energy. Also it is to be noticed that instead of showing smooth fall off, the first Born result starts to go higher TDCS value after $30 \mathrm{eV}$ ejected energy. If we increase the ejected angle $\theta_{1}=40^{\circ}$ to $\theta_{1}=60^{\circ}$ and fix the scattering angle $\theta_{2}=-5^{\circ}$ to $\theta_{2}=-10^{\circ}$ (Fig. 5), we get the similar pattern with smaller peak value than Fig. 3. Table 1 represents different components of the scattering amplitude for the ionization of hydrogen atoms by electrons of incident energy $250 \mathrm{eV}$ for various ejected electron energy $\mathrm{E} 1$, and for the scattering angle $\theta_{2}=-10^{\circ}$ and the ejection angle $\theta_{1}=40^{\circ}$. Here the scattering amplitudes $f_{e T}, f_{P T}, f_{P e}$ and $f_{P W B}$ are given in (5) and also physical origins of the findings are presented here.

In (5), for $f_{e T}, \psi_{f}^{(-)}\left(\bar{r}_{1}, \bar{r}_{2}\right)=\varphi_{\bar{p}_{1}}^{(-)}\left(\bar{r}_{1}\right) e^{\bar{p}_{2} \cdot \bar{r}_{2}}$ is the first Born amplitude in which the scattered electrons are described by a plane wave while the ejected electrons are described by a Coulomb wave. The amplitude $f_{P T}$, $\psi_{f}^{(-)}\left(\bar{r}_{1}, \bar{r}_{2}\right)=\varphi_{\bar{p}_{2}}^{(-)}\left(\bar{r}_{2}\right) e^{i \bar{p}_{1} \cdot \bar{r}_{1}}$ is similar to the first Born amplitude $f_{e T}$ except for the fact that the role of the electron and projectile is interchanged. For $f_{P e}, \psi_{f}^{(-)}\left(\bar{r}_{1}, \bar{r}_{2}\right)$ is given by $\varphi_{\bar{p}}^{(-)}(\bar{r}) e^{i \bar{P} \cdot \bar{R}} \quad, \quad$ where $\quad \bar{r}=\frac{\bar{r}_{1}-\bar{r}_{2}}{2} \quad, \quad \bar{R}=\bar{r}_{1}+\bar{r}_{2}$, $\bar{p}=\left(\bar{p}_{2}-\bar{p}_{1}\right), \quad \bar{P}=\bar{p}_{2}+\bar{p}_{1}$. In this term the projectile electron interaction is exactly treated in the final channel, where centre of mass moves as a plane wave. For $f_{P W B}$, $\psi_{f}^{(-)}\left(\overline{r_{1}}, \bar{r}_{2}\right)$ is given by $e^{i \bar{p}_{1} \cdot \bar{r}_{1}+\bar{p}_{2} \cdot \bar{r}_{2}}$ corresponding to two plane waves for the ejected electron and scattered particle. Here $N\left(\bar{p}_{1}, \bar{p}_{2}\right)$ is the normalization constant. It has been calculated numerically using (6) for the electron impact and the approximate value of $\mathrm{N}$ is nearly 1 .

Let us consider the case of Fig. 6 where we have decreased the scattering angle $\theta_{2}=-10^{\circ}$ to $\theta_{2}=-20^{\circ}$ and have increased the ejected angle $\theta_{1}=60^{\circ}$ to $\theta_{1}=80^{\circ}$, remaining the incident energy unaltered as $250 \mathrm{eV}$. In our present results, there are one small peak at about $40 \mathrm{eV}$ and one sharp peak at $72 \mathrm{eV}$ whereas, in the results of Das and Dhar [20] there were two large peaks and one small peak at about $32 \mathrm{eV}, 50 \mathrm{eV}$ and $15 \mathrm{eV}$ respectively. For this case, the first Born result appears with a binary peak at $40 \mathrm{eV}$. These results differ from other discussed results of Figures 1-5.

Next we observe that both results of Figs. 7 and 8 at incident energy of $500 \mathrm{eV}$ display the same pattern as Fig. 4.

In Fig. 9, the present result completely defines similar behavior with different peak value at about $60 \mathrm{eV}$ ejected energy in contrast to the compared results [20] with $25 \mathrm{eV}$ ejected energy. The first Born result shows steadiness after $25 \mathrm{eV}$.

Lastly, in Fig. 10, we have found two flattened peaks at about $18 \mathrm{eV}$ and $80 \mathrm{eV}$ in our present results which show somewhat similar appearance to the compared results [20] with two peaks at $10 \mathrm{eV}$ and $20 \mathrm{eV}$. i.e., the peak positions are shifted to higher ejected energy. Also, there appears a smooth peak at $40 \mathrm{eV}$ ejected energy in our first Born result.

Finally, we remark that the peak pattern of the energy spectrum as obtained from our present study is closer to the compared results [20] in some cases and again sometimes different. It may be happened because of the change of atomic state.

Table 1. Different components of the scattering amplitude for ionization of hydrogen atoms by electrons of incident energy $250 \mathrm{eV}$ for various ejected electron energy $E 1(\mathrm{eV})$, and for the scattering angle $\theta_{2}=-10^{\circ}$ and the ejection angle $\theta_{1}=40^{\circ}$, scattering taking place in a plane.

\begin{tabular}{lllll}
\hline $\mathbf{E} 1(\mathbf{e V})$ & $f_{e T}$ & $f_{P T}$ & $f_{P e}$ & $f_{P W B}$ \\
\hline 5 & $-0.9319-0.2317 \mathrm{i}$ & $-1.5164+10.9939 \mathrm{i}$ & $1.8871-1.2310 \mathrm{i}$ & -0.1613 \\
14 & $-0.6153-0.1530 \mathrm{i}$ & $-1.5155+11.0056 \mathrm{i}$ & $1.8910-1.2689 \mathrm{i}$ & -0.1065 \\
23 & $0.1192+0.0296 \mathrm{i}$ & $-1.5145+11.0206 \mathrm{i}$ & $1.9001-1.3567 \mathrm{i}$ & 0.0206 \\
32 & $0.7728+0.1921 \mathrm{i}$ & $-1.5135+11.0375 \mathrm{i}$ & $1.9082-1.4349 \mathrm{i}$ & 0.1338 \\
41 & $0.9013+0.2241 \mathrm{i}$ & $-1.5125+11.0560 \mathrm{i}$ & $1.9098-1.4504 \mathrm{i}$ & 0.1561 \\
50 & $0.4175+0.1038 \mathrm{i}$ & $-1.5113+11.0761 \mathrm{i}$ & $1.9039-1.3925 \mathrm{i}$ & 0.0723 \\
59 & $-0.3499-0.0870 \mathrm{i}$ & $-1.5101+11.0979 \mathrm{i}$ & $1.8945-1.3008 \mathrm{i}$ & -0.0606 \\
68 & $-0.8797-0.2187 \mathrm{i}$ & $-1.5087+11.1215 \mathrm{i}$ & $1.8880-1.2375 \mathrm{i}$ & -0.1523 \\
77 & $-0.8118-0.2018 \mathrm{i}$ & $-1.5073+11.1471 \mathrm{i}$ & $1.8889-1.2456 \mathrm{i}$ & -0.1406 \\
86 & $-0.1924-0.0478 \mathrm{i}$ & $-1.5057+11.1750 \mathrm{i}$ & $1.8965-1.3198 \mathrm{i}$ & -0.0333 \\
95 & $0.5577+0.1386 \mathrm{i}$ & $-1.5039+11.2056 \mathrm{i}$ & $1.9058-1.4095 \mathrm{i}$ & 0.0966 \\
\hline
\end{tabular}




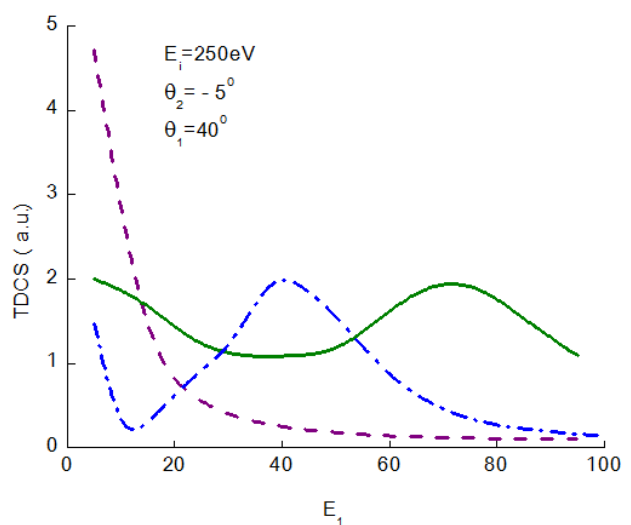

Figure 1. TDCS for ionization of hydrogen atoms by electrons is shown against ejected electron energy $E_{1}$ at $\theta_{1}=40^{\circ} \quad 250 \mathrm{eV}$ incident electron energy $E_{i}$ for scattering angle $\theta_{2}=-5^{\circ}$ and ejection angle $\theta_{1}=40^{\circ}$. Theory: Full curve: present results, Dashed curve: present first Born result and Dashed dotted curve: ground state hydrogenic results [20].

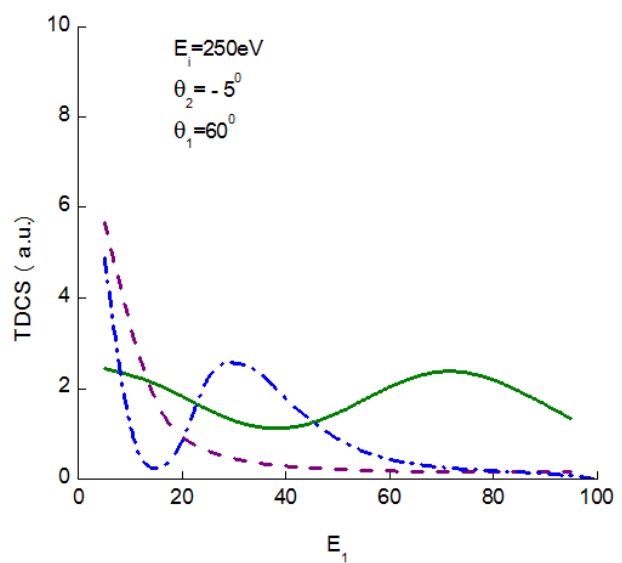

Figure 2. TDCS for ionization of hydrogen atoms by electrons is shown against ejected electron energy $E_{1}$ at $250 \mathrm{eV}$ incident electron energy $E_{i}$ for scattering angle $\theta_{2}=-5^{\circ}$ and ejection angle $\theta_{1}=60^{\circ}$. Theory: Full curve: present results, Dashed curve: present first Born result and Dashed dotted curve: ground state hydrogenic results [20].

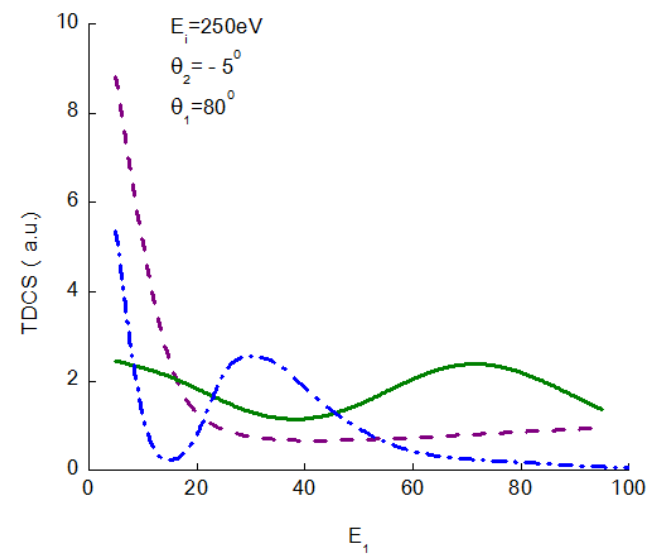

Figure 3. TDCS for ionization of hydrogen atoms by electrons is shown against ejected electron energy $E_{1}$ at $250 \mathrm{eV}$ incident electron energy $E_{i}$ for scattering angle $\theta_{2}=-5^{\circ}$ and ejection angle $\theta_{1}=80^{\circ}$. Theory: Full curve: present results, Dashed curve: present first Born result and Dashed dotted curve: ground state hydrogenic results [20].

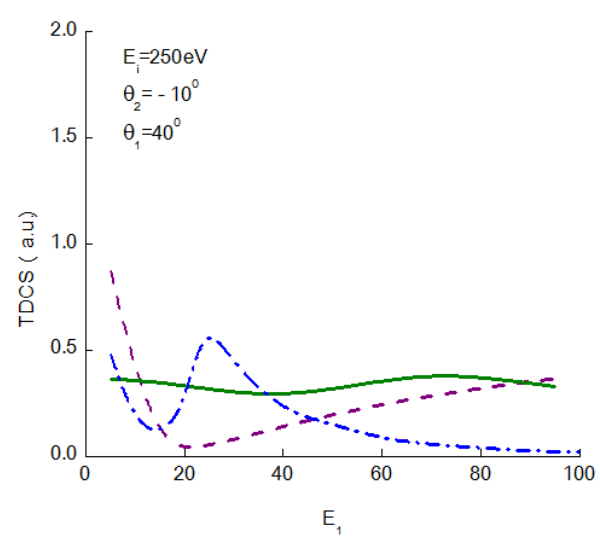

Figure 4. TDCS for ionization of hydrogen atoms by electrons is shown against ejected electron energy $E_{1}$ at $250 \mathrm{eV}$ incident electron energy $E_{i}$ for scattering angle $\theta_{2}=-10^{\circ}$ and ejection angle $\theta_{1}=40^{\circ}$. Theory: Full curve: present results, Dashed curve: present first Born result and Dashed dotted curve: ground state hydrogenic results [20].

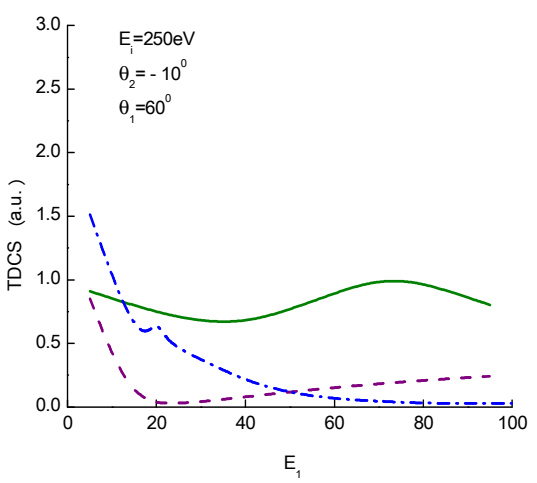

Figure 5. TDCS for ionization of hydrogen atoms by electrons is shown against ejected electron energy $E_{1}$ at $250 \mathrm{eV}$ incident electron energy $E_{i}$ for scattering angle $\theta_{2}=-10^{\circ}$ and ejection angle $\theta_{1}=60^{\circ}$. Theory: Full curve: present results, Dashed curve: present first Born result and Dashed dotted curve: ground state hydr ogenic results [20].

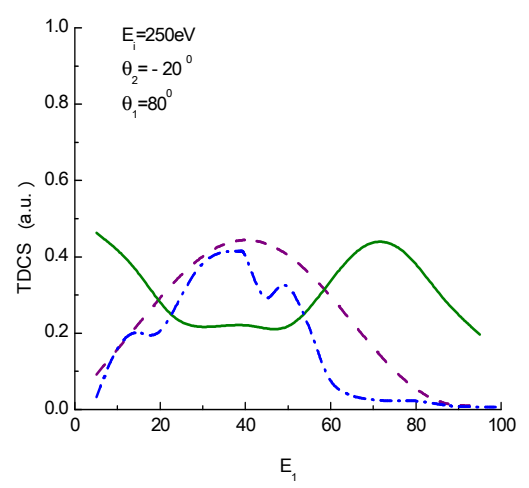

Figure 6. TDCS for ionization of hydrogen atoms by electrons is shown against ejected electron energy $E_{1}$ at $250 \mathrm{eV}$ incident electron energy $E_{i}$ for scattering angle $\theta_{2}=-20^{\circ}$ and ejection angle $\theta_{1}=80^{\circ}$. Theory: Full curve: present results, Dashed curve: present first Born result and Dashed dotted curve: ground state hydrogenic results [20]. 


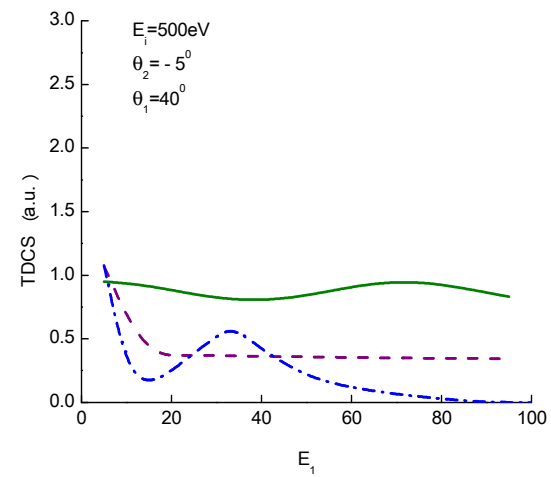

Figure 7. TDCS for ionization of hydrogen atoms by electrons is shown against ejected electron energy $E_{1}$ at $500 \mathrm{eV}$ incident electron energy $E_{i}$

for scattering angle $\theta_{2}=-5^{\circ}$ and ejection angle $\theta_{1}=40^{\circ}$. Theory: Full curve: present results, Dashed curve: present first Born result and Dashed dotted curve: ground state hydrogenic results [20].

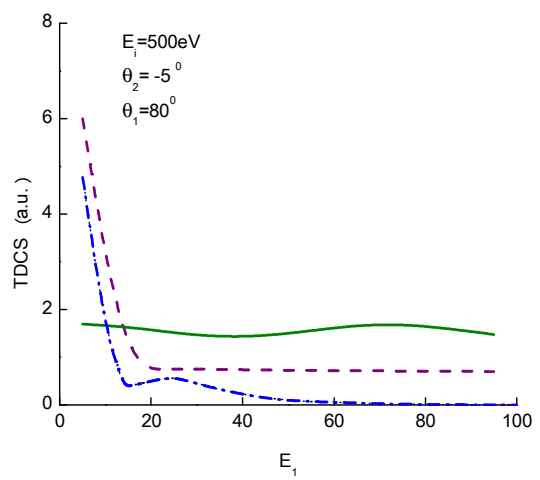

Figure 8. TDCS for ionization of hydrogen atoms by electrons is shown against ejected electron energy $E_{1}$ at $500 \mathrm{eV}$ incident electron energy $E_{i}$ for scattering angle $\theta_{2}=-5^{\circ}$ and ejection angle $\theta_{1}=80^{\circ}$. Theory: Full curve: present results, Dashed curve: present first Born result and Dashed dotted curve: ground state hydrogenic present results [20].

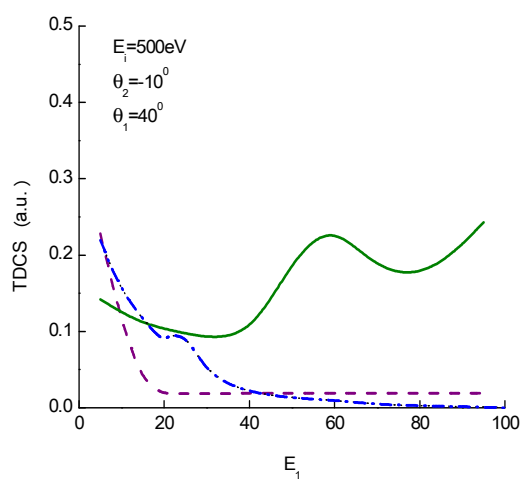

Figure 9. TDCS for ionization of hydrogen atoms by electrons is shown against ejected electron energy $E_{1}$ at $500 \mathrm{eV}$ incident electron energy $E_{i}$

for scattering angle $\theta_{2}=-10^{\circ}$ and ejection angle $\theta_{1}=40^{\circ}$. Theory: Full curve: present results, Dashed curve: present first Born result and Dashed dotted curve: ground state hydrogenic results [20].

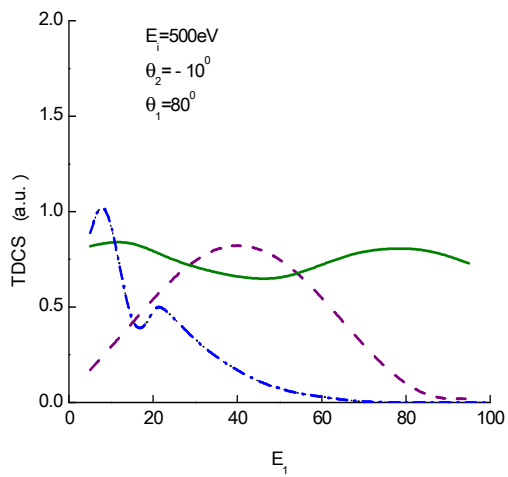

Figure 10. TDCS for ionization of hydrogen atoms by electrons is shown against ejected electron energy $E_{1}$ at $500 \mathrm{eV}$ incident electron energy $E_{i}$

for scattering angle $\theta_{2}=-10^{\circ}$ and ejection angle $\theta_{1}=80^{\circ}$. Theory: Full curve: present results, Dashed curve: present first Born result and Dashed dotted curve: ground state hydrogenic results [20].

\section{Conclusions}

The energy spectrum of scattered electrons for ionization of hydrogen atoms by electrons in metastable $2 \mathrm{P}$ state has been calculated following the multiple scattering theory of Das and Seal. The present results show very interesting binary peak features. Almost in all cases, our present study shows significant qualitative agreement with the previous hydrogenic ground state results. The present binary peak magnitudes are larger than the first Born results and the compared results of Das and Dhar. So, calculation for other related theories and experiments in the hydrogenic metastable states by electrons will be interesting.

\section{Acknowledgements}

The computational works have been performed in the Simulation Lab of the Department of Mathematics, Chittagong University of Engineering and Technology, Chittagong-4349, Bangladesh.

\section{References}

[1] J. N. Das, Nuovo Cimento B, 12, P-197, 1972.

[2] D. H. Madison and E. Merzbacher, "Atomic inner-shell processed", edited by Crazemann, B.; Academic Press, New York, USA, 1975

[3] J. N. Das and S. Chakraborty, Phys. Rev. A, 32, P-176, 1985.

[4] D. H. Jakubasa-Amundsen, Z. Phys. D, 11, P-305, 1989.

[5] H. Ehrhardt, K. Jung, G. Knoth and P. Schlemmer, Z. Phys. D, $1,3,1986$.

[6] T. D. Mark, and G. H. Dunn, "Electron impact ionization", Springer:New York, USA, 1985.

[7] F. W. Jr. Byron and C. J. Joachain, Phys. Rep., 179, P-211, 1989. 
[8] M. Brauner, J. S. Briggs and H. Klar, J.Phys. B: At. Mol. Opt. Phys., 22, P-2265, 1989.

[9] J. N. Das, Phys. Rev. A, 42, P-1376, 1990.

[10] J. N. Das and S. Seal Pramana-J. Phys., 40, P-253, 1993.

[11] J. N. Das and S. Seal, Z. Phys. D, 31, P-167, 1994a.

[12] S. Dhar, Aust. J. Phys., 49 P-937, 1996.

[13] M. Brauner, J. S. Briggs and H. Klar, J. T. Board, T. Rösel, K. Jung and H. Ehrhardt, J. Phys. B, 24, P-657, 1991.

[14] A. Lahmann-Bennani, J. Phys. B, 24, P-2401, 1991.

[15] H. Ruoff and W. Nakel, J. Phys. B, 20, P-2299, 1987.

[16] W. L. Fite and R. T. Brachmann, Phys. Rev., 112, P-1141, 1958.

[17] J. Bonfert, H. Graf and W. Nakel, J. Phys. B, 24, P-1423, 1991.
[18] N. F. Mott and H. S. W. Massey, "The theory of atomic collisions" 4rth edition; p-492, 1965.

[19] E. Schüle and W. Nakel, J. Phys. B, 15, L639, 1982.

[20] J. N. Das and Seal, S. Pramana J. Phys., 53, P-869,1999.

[21] J. N. Das and S. Seal, Phys. Rev. A, 47, P-2978, 1993a.

[22] J. N. Das and S. Dhar, J. Phys. B, 31, P-2355, 1998.

[23] S. Dhar, J. Phys. B: At. Mol. Phys., 41, P-155204, 2008.

[24] S. Dhar and N. Nahar, Open Journal of Microphysics, 4, P-46, 2014.

[25] S. Dhar and N. Nahar, Results in Physics, 4, P-170, 2014.

[26] R. R. Lewis, Phys. Rev., 102, P-537, 1956. 